\title{
HELSON SETS IN COMPACT AND LOCALLY COMPACT GROUPS
}

\section{Charles F. Dunkl and Donald E. Ramirez}

We continue our investigation (begun in [1] and [4]) of the measure space $M_{0}(G)$, where $\mathrm{G}$ denotes an infinite, nondiscrete, locally compact group, not necessarily abelian. In the present paper, we show that each measure in $M_{0}(G)$ is continuous. We further show that if $G$ is compact or metrizable, then a Helson set cannot support a nonzero measure in $\mathrm{M}_{0}(\mathrm{G}$ ) (a Helson set is a compact set $\mathrm{P}$ in $\mathrm{G}$ such that every continuous function on $\mathbf{P}$ can be extended to a function in the Fourier algebra $A(G)$ of the group $G)$.

Let $\mathrm{G}$ denote an infinite, nondiscrete, locally compact group (not necessarily abelian) with left-invariant Haar measure $m_{G}$, and let $M(G)$ denote the space of finite regular Borel measures on $G$. We use the notation and machinery developed by P. Eymard [5] as well as that in [2]. Let $\Sigma$ denote the equivalence classes of the continuous unitary representations on $\mathrm{G}$, and for $\pi \in \Sigma$, let $\mathscr{H}_{\pi}$ denote the representation space. For $\mu \in \mathrm{M}(\mathrm{G})$, we define the function $\hat{\mu}$ on $\Sigma$ by

$$
\pi \mapsto \hat{\mu}_{\pi}=\int_{\mathrm{G}} \pi(\mathrm{x}) \mathrm{d} \mu(\mathrm{x})
$$

For $\mathscr{J}^{\prime} \subset \Sigma$, let

$$
\|\mu\|_{\mathscr{P}}=\sup \left\{\left\|\hat{\mu}_{\pi}\right\|_{\infty}: \pi \in \mathscr{P}\right\}
$$

where $\left\|\hat{\mu}_{\pi}\right\|_{\infty}$ denotes the operator norm on $\mathscr{H}_{\pi}$. We define $\mathrm{C}^{*}(\mathrm{G})$ to be the completion of $L^{1}(G)$ in $\|\cdot\|_{\Sigma}$ (see $[5$, p. 187]). Let $\{\rho\}$ denote the subset of $\Sigma$ containing just the left-regular representation of $G$ on $L^{2}(G)$. Let $C^{*}(G)$ denote the completion of $\mathrm{L}^{\mathrm{l}}(\mathrm{G})$ in $\|\cdot\|_{\rho}($ see $[5, \mathrm{p} .187])$. If $\mathrm{G}$ is abelian or compact, then $\mathrm{C}^{*}(\mathrm{G})=\mathrm{C}_{\rho}^{*}(\mathrm{G})$.

If $\mu \in \mathrm{M}(\mathrm{G})$, we let $\rho(\mu)$ denote the bounded operator defined on $\mathrm{L}^{2}(\mathrm{G})$ by $\mathrm{h} \mapsto \mu * \mathrm{~h}\left(\mathrm{~h} \in \mathrm{L}^{2}(\mathrm{G})\right)$ with operator norm $\|\rho(\mu)\|_{\rho}$. Let $\mathscr{B}\left(\mathrm{L}^{2}(\mathrm{G})\right)$ denote the bounded operators on $\mathrm{L}^{2}(\mathrm{G})$. Then $\mathrm{C}_{\rho}^{*}(\mathrm{G})$ can be identified with the closure in $\mathscr{B}\left(\mathrm{L}^{2}(\mathrm{G})\right)$ of the set $\rho\left(\mathrm{L}^{\mathrm{l}}(\mathrm{G})\right)=\left\{\rho(\mathrm{f}): \mathrm{f} \in \mathrm{L}^{\mathrm{I}}(\mathrm{G})\right\}$. If $\mathrm{G}$ is abelian, then $\mathrm{C}_{\rho}^{*}(\mathrm{G})$ is isomorphic to the space $\mathrm{C}_{0}(\hat{\mathrm{G}})$ of continuous functions on the dual group $\hat{\mathrm{G}}$ that vanish at infinity; and if $G$ is compact, then $C_{\rho}^{*}(G) \cong \mathscr{C}_{0}(\hat{G})$ (see $\left.[1]\right)$.

Let $\mathrm{VN}(\mathrm{G})$ denote the von Neumann subalgebra of $\mathscr{B}\left(\mathrm{L}^{2}(\mathrm{G})\right)$ generated by the left translation operators (see [5, p. 210]). If $\mu \in \mathrm{M}(\mathrm{G})$, then $\rho(\mu) \in \mathrm{VN}(\mathrm{G})$. Furthermore, we have the inclusion $C_{\rho}^{*}(G) \subset V N(G)$. If $G$ is abelian, then $\mathrm{VN}(\mathrm{G}) \cong \mathrm{L}^{\infty}(\hat{\mathrm{G}})$; and if $\mathrm{G}$ is compact, then $\mathrm{VN}(\mathrm{G}) \cong \mathscr{L}^{\infty}(\hat{\mathrm{G}})$ (see [1]).

Let $\mathrm{B}(\mathrm{G})$ denote the linear subspace of $\mathrm{C}^{\mathrm{B}}(\mathrm{G})$ (the continuous bounded functions on $G)$ spanned by the continuous, positive-definite functions. Then $B(G)$ can be

Received June 4, 1970.

This research was supported in part by NSF contract GP-8981.

Michigan Math. J. 19 (1972). 
identified with the dual space of $C^{*}(G)$ (see $\left[5\right.$, p. 192]). For $f \in B(G)$, let $\|f\|_{B}$ denote the norm of $f$ as a linear functional on $C^{*}(G)$. Now let $A(G)$ be the closed subalgebra of $\mathrm{B}(\mathrm{G})$ generated by the continuous, positive-definite functions with compact support (see $[5, p .208])$. If $G$ is abelian, then $A(G) \cong L^{l}(\hat{G})$; and if $G$ is compact, then $\mathrm{A}(\mathrm{G}) \cong \mathscr{L}^{1}(\hat{\mathrm{G}})$ (see $[1]$ ).

The reader familiar with the abelian or compact case will not be surprised to find that the dual of $\mathrm{A}(\mathrm{G})$ is $\mathrm{VN}(\mathrm{G})$; that is, $\mathrm{A}(\mathrm{G})^{*} \cong \mathrm{VN}(\mathrm{G})$ (see $[5, \mathrm{p} .210]$ ). Also, $\mathrm{A}(\mathrm{G})$ is a $\mathrm{VN}(\mathrm{G})$-module; that is, for $\mathrm{T} \in \mathrm{VN}(\mathrm{G})$ and $\mathrm{f} \epsilon \mathrm{A}(\mathrm{G})$, we define $\mathrm{T} * \mathrm{f} \in \mathrm{A}(\mathrm{G})$ by $\langle\mathrm{T} * \mathrm{f}, \mathrm{S}\rangle=\langle\mathrm{f}, \check{\mathrm{TS}}\rangle$, where $\langle\cdot, \cdot\rangle$ denotes the pairing of $\mathrm{A}(\mathrm{G})$ with its dual space $\mathrm{VN}(\mathrm{G})$, and where $\check{\mathrm{T}}$ is given by $\langle\mathrm{g}, \check{\mathrm{T}}\rangle=\langle\check{\mathrm{g}}, \mathrm{T}\rangle$ (here $\stackrel{\mathrm{g}}{\mathrm{g}}$ denotes the element of $\mathrm{A}(\mathrm{G})$ defined by $\mathrm{g}(\mathrm{x})=\mathrm{g}\left(\mathrm{x}^{-1}\right)$; see $\left.[5, \mathrm{p} .212]\right)$. If $\mathrm{G}$ is abelian, then $\mathrm{L}^{\mathrm{l}}(\hat{\mathrm{G}})$ is an $\mathrm{L}^{\infty}(\hat{\mathrm{G}})$-module by pointwise multiplication, and if $\mathrm{G}$ is compact, then $\mathscr{L}^{\mathrm{l}}(\hat{\mathrm{G}})$ is an $\mathscr{L}^{\infty}(\hat{\mathrm{G}})$-module by coordinatewise multiplication. If $\mu \in \mathrm{M}(\mathrm{G})$ and $\mathrm{f} \in \mathrm{A}(\mathrm{G})$, then $\rho(\mu) * \mathrm{f}$ is precisely $\mu * \mathrm{f}[5, \mathrm{p} .215]$. The basic inequality that we shall need is the relation $\|\mathrm{T} * \mathrm{f}\|_{\mathrm{A}} \leq\|\mathrm{T}\|_{\mathrm{VN}}\|\mathrm{f}\|_{\mathrm{A}}(\mathrm{T} \in \mathrm{VN}(\mathrm{G}), \mathrm{f} \in \mathrm{A}(\mathrm{G}))$ (see $\left.[5, \mathrm{p} .213]\right)$.

Let $B_{\rho}(G)$ denote the functions $f \in B(G)$ for which

$$
\sup \left\{\left|\int_{G} f(x) g(x) d m_{G}(x)\right|: g \in L^{1}(G),\|g\|_{\rho} \leq 1\right\}<\infty .
$$

Then $\mathrm{B}_{\rho}(\mathrm{G})$ can be identified with the dual space of $\mathrm{C}_{\rho}^{*}(\mathrm{G})$ (see $[5, \mathrm{p} .192]$ ).

In our paper [1], we introduced the notation $\mathrm{M}_{0}(\mathrm{G})=\left\{\mu \in \mathrm{M}(\mathrm{G}): \rho(\mu) \in \mathrm{C}_{\rho}^{*}(\mathrm{G})\right\}$. This notation differs by a dash from that of one of our other papers [4]. For measures supported on compact sets, the notational differences disappear (see Proposition 3). We have chosen to define the larger space to prove a slightly stronger result. In particular,

$$
\mathrm{L}^{1} \overline{(\mathrm{G})}^{\rho} \supset \mathrm{L}^{1} \overline{(\mathrm{G})}^{\Sigma} \supset \mathrm{L}^{1}(\mathrm{G}), \quad \text { since }\|\mu\|_{\rho} \leq\|\mu\|_{\Sigma} \leq\|\mu\|,(\mu \in \mathrm{M}(\mathrm{G})) .
$$

THEOREM 1. Let $\mu \in \mathrm{M}_{0}(\mathrm{G})$. Then $\mu$ is continuous.

Proof. Define the map E: $\mathrm{M}(\mathrm{G}) \rightarrow \mathrm{C}$ by $\mathrm{E}(\mu)=\mu(\{\mathrm{e}\})(\mu \in \mathrm{M}(\mathrm{G}))$. We begin by showing that $\mathrm{E}$ is continuous on $\mathrm{M}(\mathrm{G})$ with the norm $\|\cdot\|_{\rho}$. Let $\{\alpha\}$ be a neighborhood basis of e in $G$. Let $\left\{f_{\alpha}\right\}$ be a collection of functions from $A(G)$ with the following properties: $\mathrm{f}_{\alpha}(\mathrm{e})=1,\left\|\mathrm{f}_{\alpha}\right\|_{\mathrm{A}}=1, \mathrm{f}_{\alpha}$ is positive-definite, and support $\left(\mathrm{f}_{\alpha}\right) \subset \alpha$. Now

$$
\begin{aligned}
|\mathrm{E}(\mu)|=\lim _{\alpha}\left|\int_{\mathrm{G}} \mathrm{f}_{\alpha} \mathrm{d} \mu\right| & =\lim _{\alpha}\left|\left(\mu * \mathrm{f}_{\alpha}\right)(\mathrm{e})\right| \leq\left\|\mu * \mathrm{f}_{\alpha}\right\|_{\mathrm{A}} \leq\|\rho(\mu)\|_{\mathrm{VN}}\left\|\mathrm{f}_{\alpha}\right\|_{\mathrm{A}} \\
& =\|\rho(\mu)\|_{\mathrm{VN}}=\|\mu\|_{\rho} .
\end{aligned}
$$

Since we can extend $\mathrm{E}$ to $\rho \overline{(\mathrm{M}(\mathrm{G})}$ ) $\mathrm{VN}$ (closure in $\mathrm{VN}(\mathrm{G})$ ), it is easy to see that $\mathrm{E}\left(\mu * \mu^{*}\right)=\sum_{\mathrm{x} \in \mathrm{G}}|\mu(\{\mathrm{x}\})|^{2}$, and this implies that $\mathrm{E}=0$ on $\mathrm{L}^{1}(\mathrm{G})$.

Let $\mu \in \mathrm{M}_{0}(\mathrm{G})$. Then $\mu * \mu^{*} \in \mathrm{C}_{\rho}^{*}(\mathrm{G})$, since $\mathrm{C}_{\rho}^{*}(\mathrm{G})$ is a *-algebra. Since $\mathrm{E}=0$ on $\mathrm{L}^{\mathrm{l}}(\mathrm{G}), \mathrm{E}=0$ on $\mathrm{L}^{\mathrm{l}} \overline{(\mathrm{G})} \mathrm{VN}=\mathrm{C}_{\rho}^{*}(\mathrm{G})$. Thus $\mathrm{E}\left(\mu * \mu^{*}\right)$, which is $\sum_{\mathrm{x} \in \mathrm{G}}|\mu(\{\mathrm{x}\})|^{2}$, has the value zero. Thus $\mu$ is continuous. 
COROLLARY 2. If $\mu \in \mathrm{M}(\mathrm{G})$ and $\rho(\mu)$ is unitary, then $\sum_{\mathrm{x} \in \mathrm{G}}|\mu(\{\mathrm{x}\})|^{2}=1$. Proof. Observe that $\mathrm{E}\left(\mu * \mu^{*}\right)=\mathrm{E}\left(\delta_{\mathrm{e}}\right)=1$.

Let $P$ be a compact subset of $G$. We denote by $M_{0}(P)$ the space $M(P) \cap M_{0}(G)$, and by $M_{0 \Sigma}(P)$ the space

$$
\left\{\mu \in \mathrm{M}(\mathrm{P}): \mu \in \mathrm{L}^{1} \overline{(\mathrm{G})} \Sigma \cong \mathrm{C}^{*}(\mathrm{G})\right\} .
$$

We now show that the spaces $\mathrm{M}_{0}(\mathrm{P})$ and $\mathrm{M}_{0 \Sigma}(\mathrm{P})$ coincide.

PROPOSITION 3. Let $\mathrm{P}$ be a compact subset of $\mathrm{G}$. Then $\mathrm{M}_{0}(\mathrm{P})=\mathrm{M}_{0 \Sigma}(\mathrm{P})$.

Proof. The inclusion $\mathrm{M}_{0 \Sigma}(\mathrm{P}) \subset \mathrm{M}_{0}(\mathrm{P})$ is obvious. Our results in [4] show that $\mathrm{M}_{0}(\mathrm{P}) \subset \mathrm{L}^{1} \overline{(\mathrm{U})}^{\rho}$, where $\mathrm{U}$ is some relatively compact neighborhood of $\mathrm{P}$. It remains to show that the topologies on $L^{1}(\mathrm{U})$ from the norms $\|\cdot\|_{\rho}$ and $\|\cdot\|_{\Sigma}$ are equivalent. This follows from the relation $A(G)|U=B(G)| U$.

Definition. Let $\mathrm{P} \subset \mathrm{G}$ be a compact subset of $\mathrm{G}$ such that $\mathrm{A}(\mathrm{G}) \mid \mathrm{P}=\mathrm{C}(\mathrm{P})$ (equivalently, for $\mu \in \mathrm{M}(\mathrm{P})$ suppose $\|\mu\|$ is equivalent to $\|\mu\|_{\rho}$ or $\|\mu\|_{\Sigma}$ ). We say then that $\mathbf{P}$ is a Helson set. Note that this is the same as saying that $\mathrm{B}(\mathrm{G}) \mid \mathrm{P}=\mathrm{C}(\mathrm{P})$.

We shall show (under the condition that $G$ is compact or metrizable) that no nonzero measure supported in a Helson set can be in $M_{0}(G)$.

THEOREM 4. If $\mathrm{P}$ is a Helson set in a compact group $\mathrm{G}$ and $\mu \in \mathrm{M}_{0}(\mathrm{P})$, then $\mu=0$.

Proof. As expected, the proof is modelled on the abelian analogue due to $\mathrm{H}$. Helson (see [7, p. 119]). relation

For a bounded Borel function $\phi$ on $\mathrm{P}$, we let $\mathrm{T}_{\phi}$ be defined on $\mathrm{M}_{0}(\mathrm{P})$ by the

$$
\mathrm{T}_{\phi}(\mu)=\int_{\mathrm{P}} \phi \mathrm{d} \mu \quad\left(\mu \in \mathrm{M}_{0}(\mathrm{P})\right)
$$

Now $T_{\phi}$ is a continuous linear functional on $M_{0}(P)$. Since $M_{0}(P)$ can be identified with a closed subspace of $\mathscr{C}_{0}(\hat{\mathrm{G}})$ via the Fourier transform $\mathscr{F}$, we can extend $\mathrm{T}_{\phi}$ to $\mathscr{C}_{0}(\hat{\mathrm{G}})$. Thus there exists a $\psi \in \mathscr{L}^{1}(\hat{\mathrm{G}}) \cong \mathscr{C}_{0}(\hat{\mathrm{G}})^{*}$ (see $[2$, Section 8.3.9]) such that $\mathrm{T}_{\phi}(\mu)=\operatorname{Tr}(\hat{\mu} \psi)$ for $\mu \in \mathrm{M}_{0}(\mathrm{P})$ ( $\operatorname{Tr}$ denotes the trace). Since the Fourier algebra $\mathrm{A}(\mathrm{G})$ of $\mathrm{G}$ is isomorphic to $\mathscr{L}^{\mathrm{l}}(\hat{\mathrm{G}})$ via $\mathscr{F}$, there exists an $\mathrm{f} \in \mathrm{A}(\mathrm{G}) \subset \mathrm{C}(\mathrm{G})$ with

$$
\int_{\mathrm{G}} \phi \mathrm{d} \mu=\operatorname{Tr}(\hat{\mu} \psi)=\int_{\mathrm{P}} \mathrm{fd} \mu \quad\left(\mu \in \mathrm{M}_{0}(\mathrm{P})\right)
$$

We now use the fact that $M_{0}(P)$ is a band [1]. This implies that if $\mu \in M_{0}(P)$, then so is $\operatorname{gd} \mu(\mathrm{g} \in \mathrm{C}(\mathrm{G}))$. Hence $\int_{\mathrm{P}} \phi \mathrm{gd} \mu=\int_{\mathrm{P}} \mathrm{fgd} \mu(\mathrm{g} \in \mathrm{C}(\mathrm{G}))$. It follows that $\phi \mathrm{d} \mu=\mathrm{f} \mathrm{d} \mu$.

Let $\mu \in \mathrm{M}_{0}(\mathrm{P})$, and suppose by way of contradiction that $\mu \neq 0$. By Theorem 1 , $\mu$ is continuous, and thus the support $S$ of $\mu$ is a nonempty, perfect subset of $P$. We shall show that $S$ is not extremally disconnected by proving that under our hypotheses $G$ is metrizable. 
Let $\mathscr{H}$ denote the normal subhypergroup in $\hat{\mathrm{G}}$ generated by $\left\{\alpha \in \hat{\mathrm{G}}: \hat{\mu}_{\alpha} \neq 0\right\}$, and let $\mathrm{H}=\mathscr{H}^{\perp}$ be its annihilator in $\mathrm{G}$; that is, let

$$
\mathrm{H}=\left\{\mathrm{x} \in \mathrm{G}: \mathrm{T}_{\alpha}(\mathrm{x})=\mathrm{I}_{\mathrm{n}_{\alpha}} \text { if } \alpha \in \mathscr{H}\right\}
$$

(see [6]). Now $\mathscr{H}^{\perp}$ is a closed (hence compact) normal subgroup of G, and $\mathscr{H}^{\perp \perp}=\mathscr{H}$ (where $\mathscr{H}^{\perp \perp}=\left\{\alpha \in \hat{\mathrm{G}}: \mathrm{T}_{\alpha}(\mathrm{x})=\mathrm{I}_{\mathrm{n}_{\alpha}}\right.$ for all $\left.\mathrm{x} \in \mathscr{H}^{\perp}\right\}$ ).

We now show that $\mathrm{H}$ is a finite subgroup of $\mathrm{G}$. We need the fact that if $\mathrm{H}$ is a Helson set (so that $\mathrm{A}(\mathrm{H})=\mathrm{C}(\mathrm{H})$ ), then $\mathrm{H}$ is finite. Several proofs of this are known. For example, observe that $\mathrm{A}(\mathrm{H})$ is always weakly sequentially complete [3] but that $\mathrm{C}(\mathrm{H})$ is weakly sequentially complete only if $\mathrm{H}$ is finite.

Let $\mathrm{m}_{\mathrm{H}}$ be the Haar measure on $\mathrm{H}$. Then $\mu=\mathrm{m}_{\mathrm{H}} * \mu$, and $\mu(\mathrm{E})=\mu(\mathrm{xE})$ for each Borel set $\mathrm{E}$ and each $\mathrm{x} \in \mathrm{H}$. It follows that $\mathrm{S}$ is a union of cosets of $\mathrm{H}$. This implies that $\mathrm{H}$ is a Helson set, and therefore $\mathrm{H}$ is finite.

Now $(\mathrm{G} / \mathrm{H})^{\wedge}=\mathscr{H}[6$, p. 784], and this set is countable. Thus $\mathrm{G} / \mathrm{H}$ is metrizable (as is $\mathrm{H}$ ). Thus $\mathrm{G}$ is metrizable (by the Kakutani-Birkhoff characterization of metrizable groups).

Now we can assert the existence of a point $p \in G$ that is in the closure of each of two disjoint open subsets of $S$, say $V_{1}$ and $V_{2}$. Finally, let $\chi_{1}$ be the characteristic function of $V_{1}$; we then have the required contradiction of $X_{1} d \mu=f d \mu$ (for some $f \in C(G))$.

Observe that every compact group has an infinite Helson set, provided the group contains an infinite abelian subgroup (see [7, p. 166]). This follows from the extension theorem for the Fourier algebra of a closed subgroup of a compact group [2, Section 8.6.4].

THEOREM 5. Let $\mathrm{P}$ be a Helson set in a locally compact metrizable group $\mathrm{G}$. If $\mu \in \mathrm{M}_{0}(\mathrm{P})$, then $\mu=0$.

Proof. Let $\phi$ be a bounded Borel function on P. Let $\mathrm{T}_{\phi}$ be defined on $\mathrm{M}_{0}(\mathrm{P})$ by the relation $\mathrm{T}_{\phi}(\nu)=\int_{\mathrm{P}} \phi \mathrm{d} \nu\left(\nu \in \mathrm{M}_{0}(\mathrm{P})\right)$. Now $\mathrm{T}_{\phi}$ is a continuous linear functional on $\mathrm{M}_{0}(\mathrm{P})$. Since $\mathrm{M}_{0}(\mathrm{P})$ can be identified with a closed subspace of $\mathrm{C}_{\rho}^{*}(\mathrm{G})$ via the map $\nu \mapsto \rho(\nu)$, we can extend $\mathrm{T}_{\phi}$ to $\mathrm{C}_{\rho}^{*}(\mathrm{G})$. Thus there exists an $\mathrm{f} \in \mathrm{B}_{\rho}(\mathrm{G}) \subset \mathrm{C}^{\mathrm{B}}(\mathrm{G})$ (where $\mathrm{B}_{\rho}(\mathrm{G})$ is the dual space of $\mathrm{C}_{\rho}^{*}(\mathrm{G})$ ) such that $\int_{\mathrm{P}} \phi \mathrm{d} \nu=\int_{\mathrm{P}} \mathrm{f} \mathrm{d} \nu\left(\nu \in \mathrm{M}_{0}(\mathrm{P})\right)[5, \mathrm{p} .192]$. But $\mathrm{M}_{0}(\mathrm{P})$ is a band, and therefore $\int_{\mathrm{P}} \phi \mathrm{gd} \nu=\int_{\mathrm{P}} \operatorname{fg} \mathrm{d} \nu\left(\mathrm{g} \in \mathrm{C}^{\mathrm{B}}(\mathrm{G}), \nu \in \mathrm{M}_{0}(\mathrm{P})\right)$. Thus $\phi \mathrm{d} \nu=\mathrm{fd} \nu$. Now we proceed as in the abelian and compact cases.

COROLLARY 6. If $\mathrm{G}$ is a locally compact, metrizable (nondiscrete) group, then $\mathrm{A}(\mathrm{G}) \neq \mathrm{C}_{0}(\mathrm{G})$.

Proof. Let $\mathrm{U}$ be a relatively compact open subset of $\mathrm{G}$. Then $\mathrm{L}^{1}(\mathrm{U}) \neq\{0\}$. But if $\mathrm{A}(\mathrm{G})=\mathrm{C}_{0}(\mathrm{G})$, then $\overline{\mathrm{U}}$ is a Helson set. 


\section{REFERENCES}

1. C. F. Dunkl and D. E. Ramirez, Translation in measure algebras and the correspondence to Fourier transforms vanishing at infinity. Michigan Math. J. 17 (1970), 311-319.

2. - Topics in harmonic analysis. Appleton-Century-Crofts, New York, 1971.

3. - Sidon sets on compact groups. Monatsh. Math. 75 (1971), 111-117.

4. - Homomorphisms on groups and induced maps on certain algebras of measures. Trans. Amer. Math. Soc. (to appear).

5. P. Eymard, L'algèbre de Fourier d'un groupe localement compact. Bull. Soc. Math. France 92 (1964), 181-236.

6. S. Helgason, Lacunary Fourier series on noncommutative groups. Proc. Amer. Math. Soc. 9 (1958), 782-790.

7. W. Rudin, Fourier analysis on groups. Interscience Publishers, New York, 1962.

University of Virginia

Charlottesville, Virginia 22901 
